\title{
Single-balloon enteroscopy-guided hemostasis of an anastomotic ulcer in a patient with simulta- neous enteric-drained pancreas-kidney transplant
}

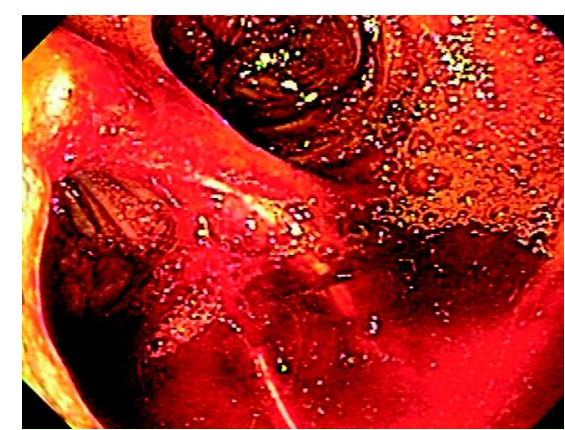

Fig. 1 Actively bleeding anastomotic ulcer.

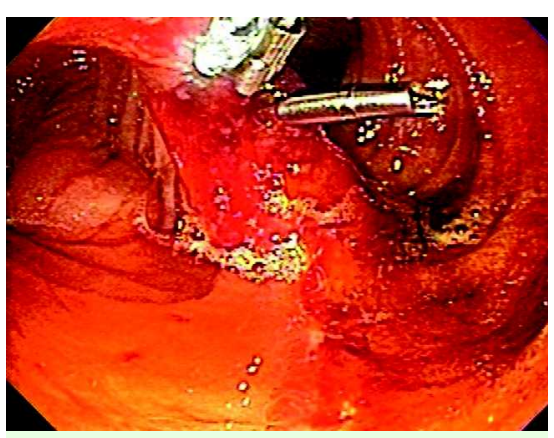

Fig. 2 Hemostasis with endoclips.

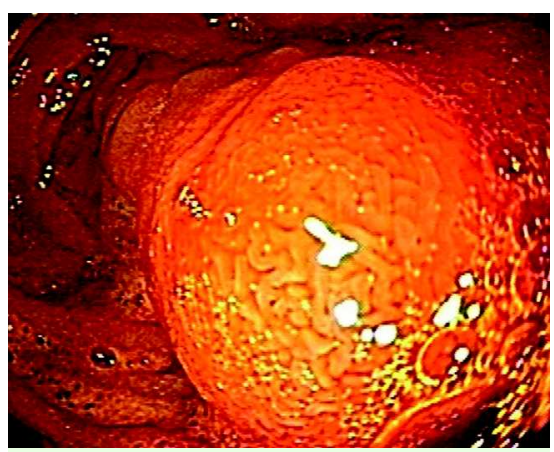

Fig. 3 Donor duodenum.

Enteric-drained pancreas transplantations have been increasing [1]. Access to the surgical anastomosis is desirable for monitoring of rejection and management of bleeding complications. A 34-year-old diabetic man with nephropathy requiring hemodialysis underwent simultaneous enteric-drained pancreas-kidney transplantation. On postoperative day 6 , he developed hematochezia. Physical examination revealed red blood in the rectum. The hematocrit declined from $35 \%$ to $23 \%$ within 24 hours. After volume resuscitation, esophagogastroduodenoscopy and colonoscopy were performed, demonstrating esophagitis, sigmoid diverticulosis, and old blood. The terminal ileum was evaluated but the surgical anastomosis was not reached. The next day, hematochezia recurred, with a drop in hematocrit from $27 \%$ to $23 \%$. A retrograde singleballoon enteroscopy (SIFQ180; Olympus Inc., Tokyo, Japan) with a disposable overtube (ST-SB1, Disposable Sliding Tube; Olympus) was performed. The duodenal stump-ileal anastomosis was reached, approximately $60 \mathrm{~cm}$ proximal to the ileocecal valve, revealing active, spurting bleeding from a visible vessel within an anastomotic ulcer ( $\bullet$ Fig. 1). Three endoclips (QuickClip2; Olympus) were placed at the bleeding site, with successful hemostasis ( $\bullet$ Fig. 2). The donor duodenum could be easily visualized ( $\bullet$ Fig. 3 ). The patient had no further bleeding episodes. The use of enteric-drained pancreas transplantation for treatment of diabetes mellitus has been limited by the frequent occurrence of complications. Repeat laparotomy is required in up to $37 \%$ of patients within 3 months of operation [1]. Anastomotic ulcers have been reported in $11.5 \%$ of patients; the risk is higher for the Roux technique [2]. Angiography may be implemented to manage such bleeding complications [3], but contrast nephropathy remains a risk. The single-balloon system was developed as an alternative to explore the small intestine [4]. This technique is particularly attractive because it can assess areas of the small intestine not evaluated by standard endoscopic instruments. Single-balloon enteroscopy may be used for monitoring pancreas rejection and management of postoperative anastomotic bleeding.

Endoscopy_UCTN_Code_TTT_1AP_2AD
S. W. de Melo Jr. ${ }^{1}$, S. Gupta ${ }^{1}$, J. Arenas², J. Sreenarasimhaiah

Digestive and Liver Diseases, University of Texas Southwestern Medical Center, Dallas, Texas, USA

2 Transplant Surgery, University of Texas Southwestern Medical Center, Dallas, Texas, USA

\section{References}

1 Reddy KS, Stratta RJ, Shokouh-Amiri MH et al. Surgical complications after pancreas transplantation with portal-enteric drainage. J Am Coll Surg 1999; 189: 305 - 313

2 Orsenigo E, Fiorina P, Dell'Antonio $G$ et al. Gastrointestinal bleeding from enterically drained transplanted pancreas. Transpl Int 2005; 18: 296- 302

3 Lopez NM, Jeon H, Ranjan D et al. Atypical etiology of massive gastrointestinal bleeding: arterio-enteric fistula following enteric drained pancreas transplant. Am Surg 2004; 70: 529-532

4 Tsujikawa T, Saitoh Y, Andoh A et al. Novel single-balloon enteroscopy for diagnosis and treatment of the small intestine: preliminary experiences. Endoscopy 2008; 40 : $11-15$

Bibliography

DOI $10.1055 / \mathrm{s}-2007-995771$

Endoscopy 2008; 40: E164

(c) Georg Thieme Verlag KG Stuttgart · New York . ISSN 0013-726X

Corresponding author

S. W. de Melo Jr., MD

University of Texas Southwestern Medical Center 4017 Prescott Avenue Apt C

Dallas

TX 75219

USA

silviomelo@sbcglobal.net 